\title{
P10: Ungleiche Stichprobenbildung beeinträchtigt den Wettbewerb im Rahmen der Transparenzprüfungen
}

\author{
Tom Schaal • Tonio Schönfelder · Jörg Klewer • \\ Joachim Kugler
}

Online publiziert: 24. Oktober 2013

(C) Springer-Verlag Wien 2013

Einleitung/Fragestellung: Die Transparenzprüfungen zur Qualitätsbeschreibung von Pflegeheimen sollen den Wettbewerb zwischen Leistungsanbietern fördern. Dabei wird zu jeder Überprüfung in Abhängigkeit der Einrichtungsgröße eine Zufallsstichprobe von 5 bis 15, jedoch nicht mehr als $10 \%$ der versorgten Bewohner gebildet. Im Rahmen dieser Studie wurde untersucht, in welchem Umfang die Vorgaben zur Stichprobenbildung erfüllt wurden und ob sich durch potentielle Abweichungen des Prüfumfangs zwischen Pflegeheimen mit gleicher Bettenanzahl infolge fehlender Chancengleichheit eine Wettbewerbsverzerrung einstellte.

Methodik/Stichprobe: Es wurden sämtliche online verfügbaren Transparenzberichte im Direktionsbezirk Dresden in die Auswertung einbezogen (Stand: 09.03.13, $N=231$, Quellen: Pflegelotse, AOK-Pflegenavigator). Es wurden deskriptive Statistiken und Häufigkeiten berechnet. Unterschiede zwischen den Vorgaben aus den Qualitätsprüfungs-Richtlinien und den gebildeten Stichproben wurden mit nichtparametrischen Tests untersucht $(p<0,05)$.

Ergebnisse: Bei 87 (37,7\%) Transparenzprüfungen wurden die Vorgaben der Stichprobenbildung in Abhängigkeit zur Einrichtungsgröße richtlinienkonform durchgeführt. Die Anzahl der einbezogenen Heimbewohner lag bei 144 (62,3\%) Prüfungen oberhalb der Vorgabewerte. Es bestand eine statistisch signifikante Abweichung zwischen der Stichprobengröße in den Transparenzberichten und den Anforderungen aus den Qualitätsprüfungs-Richtlinien $(p<0.001)$. Ein paralleler Anstieg der Abweichung war mit Zunahme der Heimplatzkapazität nicht erkennbar. Die größte Stichprobe umfasste unter den Heimen mit mehr als 50 Betten $16 \%$ der versorgten Bewohner.

Diskussion/Schlussfolgerungen: Die Varianz der Stichprobengröße bei identischer Pflegeplatzkapazität führte zu einer unterschiedlichen Repräsentativität unter den Einrichtungen innerhalb der Qualitätsberichte. Eine Wettbewerbsverzerrung infolge fehlender Chancengleichheit konnte somit nicht ausgeschlossen werden. Zur Klärung, ob homogene Stichprobengrößen $(N=9)$ durch die Novellierung der Pflege-Transparenzvereinbarung ab 2014 eine Ungleichbehandlung ausschließen, sind aufbauend auf den Ergebnissen weiterer Untersuchungen erforderlich.
T. Schaal $(\bowtie) \cdot$ J. Kugler

Lehrstuhl für Gesundheitswissenschaften/Public Health,

Medizinische Fakultät Carl Gustav Carus,

Technische Universität Dresden, Dresden, Deutschland

E-Mail: tom.schaal@gmx.de

T. Schönfelder · J. Klewer

Fakultät Gesundheits- und Pflegewissenschaften,

Westsächsische Hochschule Zwickau, Zwickau, Deutschland 